Cinémas

Revue d'études cinématographiques

Journal of Film Studies

\title{
Agencement et cinéma : la pertinence du modèle discursif en question
}

\section{Marion Froger}

Volume 10, numéro 2-3, printemps 2000

Cinéma et intermédialité

URI : https://id.erudit.org/iderudit/024813ar

DOI : https://doi.org/10.7202/024813ar

Aller au sommaire du numéro

Éditeur(s)

Cinémas

ISSN

1181-6945 (imprimé)

1705-6500 (numérique)

Découvrir la revue

Citer cet article

Froger, M. (2000). Agencement et cinéma : la pertinence du modèle discursif en question. Cinémas, 10(2-3), 13-26. https://doi.org/10.7202/024813ar

\section{Résumé de l'article}

Le présent article vise à rouvrir, après Metz, la question de la discursivité filmique, dans la perspective des études intermédiatiques, en retraçant les pistes anti-discursives que propose Deleuze pour aborder la question de l'image pensante. Il s'est agi de dégager un mode de pensée qui ne relèverait pas du discours, mais des agencements que Deleuze repère dans toute production de sens. À la lumière de cette approche, l'intermédialité a été comprise comme travail des matières d'expression entre elles, dans les termes d'une pensée du devenir, telle que la privilégie Deleuze. 


\section{Agencement et cinéma : \\ la pertinence du modèle discursif en question}

\section{Marion Froger}

\section{RÉSUMÉ}

Le présent article vise à rouvrir, après Metz, la question de la discursivité filmique, dans la perspective des études intermédiatiques, en retraçant les pistes antidiscursives que propose Deleuze pour aborder la question de l'image pensante. Il s'est agi de dégager un mode de pensée qui ne relèverait pas du discours, mais des agencements que Deleuze repère dans toute production de sens. À la lumière de cette approche, l'intermédialité a été comprise comme travail des matières d'expression entre elles, dans les termes d'une pensée du devenir, telle que la privilégie Deleuze.

\section{ABSTRACT}

This article attempts to reopen, after Metz, the question of filmic discursivity, from the perspective of intermedial studies, by retracing the anti-discursive trajectories proposed by Deleuze in his approach to the question of the thinking image. This approach derived a mode of thinking that would not fall within the province of discourse, but of agencies that Deleuze locates in all production of meaning. In light of this approach, intermediality has been understood as a working of materials of expression onto themselves, in terms of an idea of becoming, as was privileged by Deleuze.

Le "discours" est une notion bien connue des théoriciens, qui en font un usage abondant dans les études cinématographiques, littéraires, voire même musicales ou picturales. Texte et 
discours y sont étroitement liés pour décrire la production d'énoncés, leur mode d'effectuation, leur performance dans des systèmes et dispositifs "sémiotiques distincts. Ainsi, dans le champ de l'analyse filmique, discours, énoncé et texte filmiques permettent de décrire le système complet du «langage cinématographique». Cette dernière expression montre assez clatrement que la pertinence transdisciplinaire du concept de discours, importé des sciences du langage, se fonde sür une hégémonie du modèle linguistique en matière d'études sémiotiques. Or, cette hégémonie ne va pas sans poser de problèmes, dont le moindre n'est certes pas l'oubli de l'appartenance du discours à un autre domaine d'investigation et de références, que l'on dira philosophique, où se joue la question de la discursivité, c'est-à-dire de l'exercice et de l'ordonnance de la pensée dans le discours vrai.

Pour qui entend donc interroger la discursivité filmique, autrement dit l'articulation de la pensée au cinéma, la notion de discours, telle qu'importée des sciences du langage, est d'un usage plus embarrassant qu'efficace: concept purement descriptif, il ne peut en aucun cas devenir objet d'un questionnement philosophique où il s'agirait, dans la faille du discours, de cerner la possibilité d'une manifestation non discursive de la pensée; une manifestation que l'on retrouverait alors au cour de l'articulation de la pensée et du cinéma. Si en effet on tient pour acquis que le film est un discours et qu'il produit des énoncés discursifs, on ne peut que démontrer la discursivité filmique, sans être capable d'interroger la discursivité en elle-même, à l'aune de ce qui se fait au cinéma.

Gilles Deleuze est un des rares théoriciens qui renoncent à l'analyse "discursive» au cinéma. Dans L'Image-mouvement et L'Image-temps, il attaque la tradition sémiologique metzienne en se donnant pour tâche de comprendre l'articulation de la pensée en images en dehors de toute référence au modèle linguistique. Il impose ce bouleversement dans le cadre de sa démarche philosophique d'ensemble qui vise, entre autres choses ', à renouveler notre approche de l'événement (sémiotique, cognitif, affectif, etc.) à travers l'idée d'« agencement ». Il ne s'agira pas ici de rendre compte de l'«agencement» dans ce dernier degré d'abstraction qu'atteignent les développements deleuziens sur «la ma- 
chine désirante». Tout au plus tentera-t-on de souligner la pertinence du modèle cinématographique pour sa compréhension. Ces "machines désirantes" ${ }^{2}$, agencées et agençantes, ou encore ces «ensembles de voisinage homme-outil-animal-chose " (1996, p. 126) sont en effet fondamentaux dans l'érection de mondes cinématographiques. L'idée, le geste, le sentiment relèvent du désir, et par là même, appartiennent à des agencements et en produisent de nouveaux. C'est toujours une idée qui circule dans, qui émane de, qui habite ou qui hante l'espace filmique; l'image-mouvement et l'image-temps se singularisent ainsi dans les agencements qu'elles effectuent ou rendent possibles. On s'intéressera donc "aux régimes de signes" (p. 127) cinématographiques, à leur mise en œuvre d'agencements concrets où se jouent l'idée, le geste, le sentiment, hors du discours qui prétend les prendre et les signifier. L'exposé qui va suivre voudrait donc pointer quelques enjeux de l'analyse deleuzienne et les voies qu'elle ouvre, dans un contexte où les études cinématographiques se voient renouveler par l'approche intermédiatique.

\section{Du discours à l'agencement}

Si Metz assimile le cinéma à un langage, c'est, relève Gilles Deleuze dans L'Image-temps (p. 38 ssq), parce qu'il considère la constitution du film en discours, discours narratif plus précisément, comme fait premier et fondamental. Metz s'autorise alors à comparer les images à des énoncés et à fonder la discursivité filmique sur les structures syntagmatiques et paradigmatiques du modèle linguistique. Une fois cette fondation effectuée, l'analyse du discours filmique devient possible et le récit passe du statut de fait premier à celui de mode discursif parmi d'autres. Le film, en effet, de par sa structure syntagmatique, pourra alors se décliner sur tous les modes discursifs, qu'il soit narratif, descriptif, poétique ou réflexif. Des études pourront naître sur les formes syntagmatiques privilégiées par le documentaire, le film anthropologique, la fiction classique, etc.

Pour Deleuze, la production de sens ne se joue pas dans des structures et des codes discursifs sous-jacents. Son modèle ne sera plus linguistique ni son point de vue structural; l'un sera matériel et l'autre, immanent. Ainsi, et pour le dire en une 
formule, il ne sera plus question de discours, mais d'agencement. Nous nous frotterons à la matière signalétique du cinéma qui se différencie en images-mouvements et en images-temps dans une prolifération de signes, et où se forment des compositions (organique ou cristalline), qui ne reposent sur aucune structure discursive. On verra dans cette matière signalétique une projection du chaos, du virtuel, que Deleuze veut constituer comme objet de pensée:

\begin{abstract}
On définit le chaos moins par son désordre que par la vitesse infinie avec laquelle se dissipe toute forme qui s'y ébauche. C'est un vide qui n'est pas un néant, mais un virtuel, contenant toutes les particules possibles et tirant toutes les formes possibles qui surgissent pour disparaître aussitôt, sans consistance ni référence, sans conséquence. C'est une vitesse infinie de naissance et d'évanouissement. Or la philosophie demande comment garder les vitesses infinies tout en gagnant de la consistance, en donnant une consistance propre au virtuel (1991, p. 112).
\end{abstract}

Pour donner une consistance propre au virtuel, la philosophie s'aide de l'art; elle s'appuie sur le cinéma, dans la mesure où ses images sont moins la représentation des choses que leur précipitation dans une solution où entrent toutes les composantes de l'image. Deleuze nous invite donc à effectuer un premier saut hors du paradigme de la reproduction et de la ressemblance pour analyser les rapports de la pensée et de l'image.

\title{
Énonciation: pensée discursive et pensée cristalline
}

Dans L'Image-mouvement et L'Image-temps, Deleuze utilise le dispositif cinématographique pour rompre les noces de la pensée et de la discursivité, et tenter d'analyser la première hors du carcan de la seconde. Il poursuivra sa réflexion dans Qu'est-ce que la philosophie? en exploitant philosophiquement les concepts forgés dans les deux autres livres. Le carcan discursif dont il est question ici a pour armature les développements logicodéductifs que suit la pensée pour s'articuler. En eux, Deleuze ne voit aucune forme nécessaire et suffisante de l'énonciation de la pensée: 
Plutôt qu'un enchaînement de propositions, il vaudrait mieux dégager le flux du monologue intérieur, ou les étranges bifurcations de la conversation la plus ordinaire, en les séparant eux aussi de leurs adhérences psychologiques et sociologiques, pour pouvoir montrer comment la pensée produit quelque chose d'intéressant, quand elle accède au mouvement infini qui la libère du vrai comme paradigme supposé et reconquiert une puissance immanente de création (1991, p. 133).

Pour Deleuze, la pensée est ce qui affronte le chaos et l'infini. Or, face au chaos et à l'infini, il distingue trois attitudes: philosophique, artistique et mathématique. Par cette distinction, il rapproche philosophie et art comme il oppose philosophie et mathématiques. La philosophie approche les événements, les mathématiques approchent les états de choses. La première attitude crée des concepts et la deuxième, des fonctifs. Le mode d'énonciation privilégié par la première sera le monologue intérieur et par la deuxième, celui de la fonction mathématique.

Mais d'un autre côté, donc, le lien entre le cinéma et la philosophie se resserre: le monologue intérieur prend corps dans une matière signalétique définie comme une "[...] masse plastique, une matière a-signifiante et a-syntaxique, une matière non linguistiquement formée, bien qu'elle ne soit pas amorphe et soit formée sémiotiquement, esthétiquement, pragmatiquement " (1985, p. 44). Ce monologue intérieur apparaît dans une composition cristalline, s'actualise dans une image-cristal. Si les cinéastes pensent avec des images-cristal au lieu de concepts, en retour, la structure cristalline se retrouvera aussi dans le concept philosophique, dessinant ainsi le mouvement de la pensée hors de toute référence discursive, car la structure cristalline peut envelopper "les ruptures", "les catastrophes", "les bifurcations" par lesquelles passe la pensée et qui lui donnent, si elle sait les rendre, le pouvoir d'appréhender le temps.

L'image-cristal en tant qu'image pensante décrira ainsi un mode de manifestation du concept qui se libère complètement du carcan logico-déductif, de l'enchaînement de propositions. Les images pensantes du cinéma seront des concepts en acte, soit le mode idéal d'expression de l'événement qui est, Deleuze 
ne se lasse pas de le répéter, l'objet du concept, par opposition à l'état de choses qui est l'objet des fonctions mathématiques. Il serait erroné de percevoir l'image-cristal comme la description d'un état de choses. Elle est, dans toutes les virtualités qu'elle comporte, qu'elle ne cesse de déplier, le lieu de passage d'un événement, de quelque nature qu'il soit:

La philosophie ne cesse par concepts d'extraire de l'état de choses un événement consistant, un sourire sans chat en quelque sorte, tandis que la science ne cesse par fonctions d'actualiser l'événement dans un état de choses, une chose ou un corps référable (1991, p. 120).

La cristallisation est d'ailleurs le procédé même de l'abstraction, remarque Christine Buci-Glucksmann :

[...] l'abstraction cristalline. Sorte d'image-pensée au sens de Walter Benjamin, le cristal comme modalité de l'événement, du temps et de l'image renvoie à toute une esthétique du virtuel comme force. Ainsi de ces "cristaux de temps" propres au cinéma d'Ophüls, Welles ou Resnais, avec leur image biface, réelle et virtuelle, pratiquant "une coalescence" du présent et du passé, qui donne à voir le temps (1998, p. 96).

Dans la perspective deleuzienne, le discours n'est donc plus un mode d'énonciation privilégié de la pensée. Comme on l'a vu, pour penser «la pensée et le cinéma» — c’est le titre du septième chapitre de L'Image-temps —, il faut se libérer non seulement du discours mais aussi de la discursivité, qui prétend décrire les opérations de pensée dans leur coïncidence avec l'énonciation discursive, elle-même tributaire du modèle logicodéductif qui prime en mathématiques. Nous touchons ici au cœur du sujet. C'est en effet le seul moyen pour Deleuze de comprendre comment "[...] les cinéastes pensent avec des images-mouvements, et des images-temps, au lieu de concepts " (1983, p. 7-8) sans retomber dans le glissement metzien qui fait du film une forme parmi d'autres de discours. Le renversement deleuzien consiste à proposer à la philosophie, "créatrice" de concepts, non pas le modèle mathématique, mais le modèle artistique et plus particulièrement peut-être le modèle cinémato- 
graphique. Ce renversement est bien sûr au cœur de l'analyse deleuzienne du cinéma.

\section{Articulation : du logico-déductif à l'impuissance discursive. Rôle du montage comme agencement}

Deleuze renoncera à toute analyse qui arrime le montage au modèle logico-déductif, c'est-à-dire aux analyses qui mettent de l'avant l'enchaînement et la linéarité de la suite des plans cinématographiques pour les rendre semblables aux chaînes linguistiques des énoncés textuels. Une description entièrement renouvelée du montage va donc être fort utile pour décrire le rapport de la pensée et du cinéma. C'est en effet le propos de Deleuze dans le septième chapitre de L'Image-temps, où le philosophe s'attarde sur des agencements d'images qui permettent à la pensée de «[...] produire quelque chose d'intéressant»(1991, p. 133). Deleuze donne deux exemples, le cinéma d'Eisenstein et celui que conçoit Antonin Artaud. C'est à ce dernier que Deleuze emprunte le topos de l' "impuissance de la pensée ${ }^{3}$ " qui serait prise en charge par le cinéma. Le propre de la pensée en images serait en effet de penser l'impuissance à penser comme impossibilité de l'articulation dans un discours. C'est fondamentalement parce que l'image assume cette impuissance et la livre telle quelle qu'elle se fait pensée: elle passe par-dessus le discours pour rejoindre «la réalité intime du cerveau ${ }^{4}$ ». Passer par-dessus le discours signifie concrètement au cinéma: renoncer à produire du sens par enchaînement ou association d'images et en prêtant allégeance au modèle du vrai. Un modèle du vrai qui a plusieurs figures: celle du vraisemblable en fiction, par exemple, et celle de l'objectivité, dans le domaine du documentaire. Construire $\mathrm{du}$ vraisemblable, distinguer l'objectif du subjectif, c'est justement ce que permet de ne pas faire l'image cinématographique. L'image, au-delà du vraisemblable et du distinguable, figure, dans la singularité de ses agencements concrets, une impuissance à penser qui est au cœur de la pensée elle-même.

L'impuissance discursive fait aussi le lit du problématique: le cinéma se fait par là image de la pensée. On se souvient du Discours de la méthode de Descartes, où le savoir se construit par étapes, dans le raisonnement, où il n'est jamais question de 
choix, mais toujours de jugement. Le choix sans connaissance n'est qu'un pis-aller de la morale provisoire. Deleuze oppose alors la nécessité du choix avant tout savoir et dresse ainsi comme premier pilier de la pensée non discursive la question du problématique. Le problématique n'est pas cette zone qui sépare l'ignorance de la connaissance et qu'il faut traverser sous la conduite de la raison et de ses énoncés. Le problématique se dresse comme ce qui demeure sans réponse, et qui, parce que sans réponse, impose un choix. C'est d'ailleurs " [...] le thème le plus haut du film chez Dreyer, Bresson, ou Rohmer : l'identité de la pensée avec le choix comme détermination de l'indéterminable " (1985, p. 231).

C'est un cinéma qui invente aussi la coupure irrationnelle: l'enchaînement logico-déductif est écarté au profit d'un dehors censé travailler entre les chaînes d'images, et contre leur association, leur unité. Ce dehors ou domaine de l'a-discursif par excellence coupe les images les unes des autres. Tout se passe alors entre les images, dans les interstices, "interstice" faisant ici écho "à intervalle", apparu dès l'analyse de l'image-mouvement, et au plus petit circuit possible entre l'actuel et le virtuel qui définit l'image-cristal.

Le tout se confond alors avec ce que Blanchot appelle la force de "dispersion du Dehors", ou "le vertige de l'espacement": ce vide qui n'est plus une part motrice de l'image, et qu'elle franchirait pour continuer, mais qui est la mise en question radicale de l'image (tout comme il y a un silence qui n'est plus la part motrice ou la respiration du discours, mais sa mise en question radicale); le faux raccord, alors, prend un nouveau sens en même temps qu'il devient la loi (1985, p. 235).

La coupure irrationnelle est une atteinte à la linéarité et à l'enchaînement discursif. Deleuze diagnostique aussi cette atteinte dans le recours à l'écran noir et à l'écran blanc:

Cette nouvelle valeur de l'écran noir ou blanc nous semble correspondre aux caractères analysés précédemment: d'une part, ce qui compte n'est pas l'association des images, la manière dont elles s'associent, mais l'interstice entre deux images; d'autre part, la coupure 
dans une suite d'images n'est plus une coupure rationnelle qui marque la fin de l'une ou le début d'une autre, mais une coupure dite irrationnelle qui n'appartient ni à l'une ni à l'autre, et se met à valoir pour elle-même (1985, p. 260).

\section{Devenir : la montée de la matière}

Nous aimerions nous permettre ici de plier la perspective intermédiatique pour rejoindre l'approche deleuzienne, où il n'est, on le sait, finalement jamais question d'intermédialité; cette pliure se fera sur la question du rôle de la matière dans les contenus de pensée. Ce que j'appellerai le point de vue de la matérialité, chez Deleuze, traite à la fois des matériaux et des composantes de l'image cinématographique, et plus généralement, de toute œuvre d'art. Matière et matériaux, c'est-à-dire la pâte de couleur du peintre, la glaise du sculpteur, les corps des acteurs, la lumière de l'opérateur; composantes, soit les textes, les images et les sons. On comprend dès lors qu'il ne s'agit plus d'étudier l'incidence du média sur le discours, mais l'exploration d'une puissance expressive a-discursive qu'il faut se donner les moyens de comprendre en réinvestissant le point de vue de la matière. Et c'est dans ce contexte que surgit à nouveau le concept d'agencement.

Dans un premier temps, donc, Deleuze définit une expressivité de la matière: la matière est dotée d'une puissance expressive que la pensée cristallise. Cette puissance expressive, il l'analyse de deux manières. Premièrement, il décrit une montée de la matière dans la sensation et une descente de la sensation dans la matière :

On peint, on sculpte, on compose, on écrit avec des sensations. On peint, on sculpte, on compose, on écrit des sensations. Les sensations comme percepts ne sont pas des perceptions qui renverraient à un objet (référence) : si elles ressemblent à quelque chose, c'est d'une ressemblance produite par leurs propres moyens, et le sourire sur la toile est seulement fait de couleurs, de traits, d'ombre et de lumière. Si la ressemblance peut hanter l'œuvre d'art, c'est parce que la sensation ne se rapporte qu'à son matériau: elle est le percept ou 
l'affect du matériau même, le sourire d'huile, le geste de terre cuite, l'élan de métal, l'accroupi de la pierre romane et l'élevé de la pierre gothique. Et le matériau est si divers dans chaque cas (le support de la toile, l'agent du pinceau ou de la brosse, la couleur dans le tube) qu'il est difficile de dire où il finit et où commence la sensation, en fait; la préparation de la toile, la trace du poil de pinceau font évidemment partie de la sensation, et bien d'autres choses en deçà. [...] La sensation ne se réalise pas dans le matériau sans que le matériau ne passe entièrement dans la sensation, dans le percept ou l'affect. Toute la matière devient expressive (1991, p. 156-157).

Une matière expressive permet dès lors de poser le passage d'un matériau à l'autre, comme principe d'une entr'expression des choses, selon la belle formule de Leibniz. Bon nombre d'artistes contemporains agissent d'ailleurs en médiateurs de cette entr'expression ${ }^{5}$. On dira qu'à chaque fois, un agencement concret, constitué d'éléments matériels multiples, répond à un autre agencement concret, permettant ainsi la migration du percept libéré de son ancrage psychologique. On se souvient que cette conservation des percepts, des affects, indépendamment de ceux qui les perçoivent et les ressentent, est, pour Gilles Deleuze, le fondement $\mathrm{du}$ geste artistique. Or, dans ce que nous avons ici convenu d'appeler la nouvelle sphère intermédiatique, les exemples d'une conservation dans la matière, par la matière, indépendamment de tout paradigme de la ressemblance (l'image, le son ne sont pas faits pour ressembler aux sentiments, aux perceptions, aux états de choses) se multiplient. La manipulation conjointe de médias différents favorise l'élévation de nouvelles coïncidences entre les percepts, bien loin des perceptions communes qui attestent d'ordinaire l'existence des choses. Dans L'Imagetemps, Gilles Deleuze voyait dans le cinéaste cette figure de l'artiste qui est capable de nous redonner la croyance au monde, non parce qu'il passe par des attestations de ressemblance, mais parce qu'il invente de tout autres circuits; car l'image cinématographique, trop souvent tirée du côté de l'analogique de par son origine photographique, ne vaut pas comme attestation visuelle, dans un monde surmédiatisé, où l'image est aplanie (télévi- 
suelle) ou évidée (électronique) de toute matière. La croyance au monde doit enprunter d'autres chemins pour aboutir, dans l'entrelacs de correspondances non motivées. Concrètement parlant, la spécificité du régime de signes cinématographiques consisterait alors à tracer de vastes cercles inscrits dans la matière même de l'image, autour de chaque événement physique, sémiotique, cognitif, affectif, etc., cercles où se construisent des agencements concrets qui pourraient donner naissance, pour reprendre l'exemple de Deleuze, à quelque "steppe anorexique ${ }^{6}$ ", dans un seul regard, une seule parole de femme (et qui par là même, serait capable de nous redonner la signification de l'anorexie $^{7}$, à la confluence de plusieurs flux : flux alimentaire, flux de langage, flux géographique).

Dans un deuxième temps, Deleuze décrit des agencements ou compositions, où les composantes s'affectent les unes les autres : cette affection peut prendre la forme d'une transformation des effets de sens du visuel par l'arrivée du sonore. Par exemple, dans L'Image-temps, Deleuze oppose le cinéma muet au cinéma parlant, non pas en analysant ce que le son apporte de plus à l'image, mais la transformation de l'image elle-même, de sa signification comme composé visuel-sonore: pour Deleuze, l'image muette naturalisait ce qu'elle montrait, même les attitudes humaines, les visages, les décors, alors que l'arrivée du son, de la parole dénaturalise cette image qui se charge alors «de tout un domaine qu'on pourrait appeler des interactions humaines ", corrélats des actes de parole et de silence (p. 294).

C'est donc dans un contexte intermédiatique qu'il est permis d'étudier des agencements de sons et d'images, qui, en se composant, acquièrent de nouvelles qualités. Quand le son se visualise, l'image devient lisible, par exemple:

Ce que Philippon dit à propos de Beyrouth la rencontre, d'Alaouie, vaut pour tout cinéma parlant, digne de ce nom darıs sa différence essentielle avec le théâtre: «On voit véritablement la parole se frayer un chemin au travers des ruines [...]. [L'auteur] a filmé la parole comme quelque chose de visible, comme une matière en mouvement $\%$. Alors apparaît le renversement qui tend à se produire dans le parlant, par rapport au muet: au lieu d'une image vue et d'une parole lue, l'acte de parole 
devient visible en même temps qu'il se fait entendre, mais aussi l'image visuelle devient lisible, en tant que telle, en tant qu'image visuelle où s'insère l'acte de parole comme composante (1983, p. 303).

Le concept de devenir, essentiel à l'analyse deleuzienne, caractérise une forme de rapport entre deux choses hétérogènes qui s'oppose au concept d'imitation et de représentation. Un son qui se visualise dans une image cinématographique ne signifie pas qu'il se substitue à une image, ou qu'il donne à voir, cela signifie surtout qu'en se transformant au contact de l'image, il affecte celle-ci au point qu'elle se transforme à son tour, et devient lisible. "Devenir" désigne ce qui affecte la parole quand elle est prise dans l'image cinématographique (comme ailleurs Deleuze décrira ce qui affecte le son quand il est pris dans une composition musicale) et réciproquement ce qui affecte l'image au contact de ce son visualisé. Il s'agit là d'un événement, d'une rencontre, d'un agencement créateur.

Ce phénomène intermédiatique de visibilité du son et de lisibilité de l'image que nous cherchons à pointer ici, dans ces agencements ou composés visuels et sonores que sont les images cinématographiques, peut se définir comme suit: un devenirtexte des images bien loin de toute idée de transposition, traduction ou équivalence. L'intermédialité apparaît dès lors comme un champ de devenir, où les changements, les mouvements vont en se multipliant, en s'intensifiant, en se complexifiant.

C'est au contraire parce que la parole cesse de faire voir et d'être vue, c'est parce qu'elle devient indépendante de l'image visuelle, que l'image visuelle pour son compte accède à la nouvelle lisibilité des choses, et devient une coupe archéologique ou plutôt une coupe stratigraphique qui doit être lue [...]. C'est un nouveau sens de "lisible" qui apparaît pour l'image visuelle, en même temps que l'acte de parole devient pour luimême image sonore autonome (1983, p. 320).

Coupe archéologique de ce fameux chaos dont Gilles Deleuze voulait faire l'objet de la philosophie, duquel surgissent les événements de pensée, de sensations qui font toute l'épaisseur sémantique de l'image cinématographique. 


\section{Conclusion}

L'intermédialité entendue comme nouvelle approche de la production de sens, comme nouvelle approche de nos modes de penser et de créer, envelopperait donc ce parcours deleuzien qui passe par l'abandon du modèle linguistique et l'exploration du non-discursif, l'adoption du point de vue de la matérialité et la considération d'agencements où s'esquissent, se nouent, se libèrent des formes de devenir, des événements auxquels la pensée veut donner consistance. L'intermédialité n'est pas un champ d'expérimentation des "nouveaux médias", mais un long, patient ou parfois fulgurant travail d'approche du chaos. L'agencement deleuzien se révèle un concept clé pour des études intermédiatiques. Il pointe la nouveauté non pas dans les juxtapositions ou associations du texte, de l'image et du son permises par les nouveaux médias, mais dans les devenirs de ces composantes. Conséquemment, il invite à produire des agencements de concepts dont il s'agira aussi d'éprouver le devenir..., comme Deleuze l'a fait, par exemple, avec son concept de signe qui passe, et les sémioticiens en savent quelque chose, par toutes sortes d'affections particulières, épinglées sous les vocables nomades de "sonsigne", "opsigne", "mnémosigne ", "onirosigne", "hyalosigne", "chronosigne" ou encore, "lectosigne" (1985, p. 358).

Université de Montréal

\section{NOTES}

1 Bien malin celui qui saurait résumer la portée philosophique de l'œuvre de Gilles Deleuze en une seule phrase! Ce n'est bien sûr pas ici notre intention.

2 Dans Dialogues, Gilles Deleuze et Claire Parnet écrivent: "Machines abstraites ou corps sans organes, c'est le désir. Il y en a de beaucoup de sortes, mais ils se définissent par ce qui se passe sur eux, en eux: des continuums d'intensité, des blocs de devenir, des émissions de particules, des conjugaisons de flux. Or ce sont ces variables (quels continuums? quels devenirs, quelles particules, quels flux, quels modes d'émissions et de conjugaisons?) qui définissent des "régimes de signes" "(Paris: Flammarion, 1996, p 127).

3 «Ce que le cinéma met en avant, ce n'est pas la puissance de la pensée, c'est son impouvoir, et la pensée n'a jamais eu d'autre problème" (Gilles Deleuze, L'Imagetemps, Paris: Minuit, 1985, p. 216).

4 «Il s'agit bien, comme dit Artaud, de "rejoindre le cinéma avec la réalité intime du cerveau", mais cette réalité intime n'est pas le Tout, c'est au contraire une fissure, 
une fêlure. Tant qu'il croit au cinéma, il le crédite, non pas du pouvoir de faire penser le tout, mais au contraire d'une "force dissociatrice" qui introduirait une "frgure de néant", un "trou dans les apparences". Tant qu'il croit au cinéma, il le crédite, non pas du pouvoir de revenir aux images, et de les enchaîner suivant les exigences d'un monologue intérieur et le rythme des métaphores, mais de les "désenchaîner", suivant des voix multiples, des dialogues internes, toujours une voix dans une autre voix. Bref, c'est l'ensemble des rapports cinéma-pensée qu'Artaud bouleverse : d'une part il n'y a plus de tout pensable par montage, d'autre part il n'y a plus de monologue intérieur énonçable par image" (Gilles Deleuze, L'Image-temps, Paris: Minuit, 1985, p. 218).

5 Le volet culturel du colloque du CRI, avec la collaboration des "Mercredi de la veille" du Musée d'art contemporain de Montréal, a permis aux participants d'assister à une table ronde réunie par l'artiste interactif montréalais Luc Courchesne sur le thème de "l'art comme exercice de fabrication de preuves". À l'ère de l'électronique, c'est-à-dire du pouvoir de transcrire, convertir et manipuler tout le spectre des perceptions humaines, les artistes étaient invités à penser leur rapport au monde et le monde qu'ils attestent, à travers leurs pratiques artistiques. Ont participé à cette table ronde Robert Racine, écrivain et compositeur dont le dernier projet consista à écrire la partition musicale du dictionnaire de la langue française, Atau Tanaka, musicien qui compose à partir de la pulsation des corps, et Nicolas Reeves, dont la dernière réalisation est une harpe à nuages.

6 "Le même plan sera pris et repris à des niveaux très différents, suivant que les choses se passent sur "mon" corps, sur un corps social, sur un corps géographique (mais mon corps aussi est une géographie, ou un peuple, ou des peuples). [...] Il peut y avoir un désert du corps hypocondriaque, une steppe du corps anorexique, une capitale du corps paranoïaque [...] Vous êtes toujours un agencement pour une machine abstraite, qui s'effectue ailleurs dans d'autres agencements. Vous êtes toujours au milieu de quelque chose, plante, animal ou paysage" (Gilles Deleuze et Claire Parnet, Dialogues, Paris : Flammarion, 1996, p. 134 et 135).

7 " [...] et surtout conjugaison de flux (le flux alimentaire entre en rapport avec un flux vestimentaire, un flux de langage, un flux de sexualité: tout un devenir-femme moléculaire chez l'anorexique, qu'il soit homme ou femme). C'est ce que nous appelons un régime de signes" (Gilles Deleuze et Claire Parnet, Dialogues, Paris : Flammarion, 1996, p. 133).

\section{OUVRAGES CITÉS}

Buci-Glucksmann, Christine. "Les cristaux de l'art: une esthétique du virtuel». Rue Descartes, $n^{\circ} 20$ (1988), p. 95-111.

Deleuze, Gilles et Claire Parnet. Dialogues. Flammarion, Paris, 1996.

Gilles Deleuze et Félix Guattari. Qu'est-ce que la philosophie? Paris: Minuit, 1991.

Deleuze, Gilles. L'Image-temps. Paris: Minuit, 1985.

Deleuze, Gilles. L'Image-mouvement. Paris : Minuit, 1983. 